\section{An alternative care pathway for suspected seizures in pre-hospital care: a service evaluation}

\section{Jon Mark Dickson*}

The Academic Unit of Primary Medical Care, University of Sheffield j.m.dickson@sheffield.ac.uk

\section{Gregg H. Rawlings}

Academic Neurology Unit, University of Sheffield ghrawlings1@sheffield.ac.uk

\section{Richard A. Grünewald}

Department of Neurology, Royal Hallamshire Hospital r.a.grunewald@sheffield.ac.uk

\section{Kate Miles}

Department of Neurology, Royal Hallamshire Hospital kate.miles@sth.nhs.uk

\section{Carina Mack}

Department of Neurology, Royal Hallamshire Hospital carina.mack@sth.nhs.uk

\section{Thomas Heywood}

Yorkshire Ambulance Service thomas.heywood@yas.nhs.uk

\section{Markus Reuber}

Academic Neurology Unit, University of Sheffield m.reuber@sheffield.ac.uk
British Paramedic Journal 2017, vol. 2(2) 22-28

(c) The Author(s) 2017 ISSN 1478-4726

Reprints and permissions: info@class.co.uk

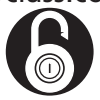

OPEN ACCESS

The BPJ is the journal of the

College of Paramedics: www.collegeofparamedics.co.uk

\begin{abstract}
Introduction - An uncomplicated, self-limiting epileptic seizure in a patient with an established diagnosis of epilepsy usually requires only first aid, but in the UK it is estimated that $75 \%$ of these patients are transported to hospital and many are discharged without review or follow-up with an epilepsy specialist. Alternative care pathways have the potential to reduce unnecessary conveyance to hospital and to improve rates of epilepsy specialist follow-up, and thereby increase the quality and cost effectiveness of care.

Methods - A service evaluation of a new alternative care pathway in a regional ambulance service in the UK. The alternative care pathway allowed paramedics to refer eligible patients to an epilepsy specialist nurse service.
\end{abstract}

* Corresponding author:

Jon Mark Dickson, The Academic Unit of Primary Medical Care, The Medical School, The University of Sheffield, Room 215, 2nd Floor, Samuel Fox House, Northern General Hospital, Herries Road, Sheffield S5 7AU, UK. 
Results - The ambulance service managed 3964 suspected seizure incidents in the study period (1 July 2015-31 May 2016), of which 22.5\% (891/3964) were potentially eligible for the alternative care pathway. Of the potentially eligible incidents, 9.8\% (87/891) were referred. The 87 incidents were generated by 74 individual patients. A total of $97.3 \%(71 / 73)$ patients were contacted within the target time of five days, the average time taken for each phone call was 10 minutes and the average additional work load generated by each call was 10 minutes. There was a positive outcome in $55 \%$ (48/87) of incidents.

Conclusions - An alternative care pathway for people after a suspected seizure has the potential to safely reduce rates of transport to hospital and to improve care for people with epilepsy. However, paramedics in our study used the alternative care pathway for only a small proportion of those patients who were potentially eligible. Further research is required to develop tools to support paramedics to confidently identify patients that are suitable for management without transport to hospital.

\section{Keywords}

accident \& emergency medicine; epilepsy; health economics; health services administration \& management; internal medicine; neurology

\section{Introduction}

A single, uncomplicated, self-limiting, epileptic seizure in a patient with an established diagnosis of epilepsy usually only requires first aid, but suspected seizures are dramatic and understandably frighten observers, who frequently call emergency pre-hospital services (Ridsdale, Virdi, Noble, \& Morgan, 2012). Epilepsy is an ambulatory care sensitive condition (ACSC) (Bardsley, Blunt, Davies, \& Dixon, 2013), and sub-optimal ambulatory care (also known as routine or scheduled care) leads to unnecessary demand for emergency care (Manjunath et al., 2012). Precise estimates vary, but in England (population 52.96 million, 42.96 million adults) seizures give rise to approximately 211,000 calls to ambulance services per year (3.3\% of all emergency calls) (Dickson et al., 2015). It is estimated that there are 60,000 seizure-related emergency department (ED) attendances per year (2-3\% of all attendances) (Dixon, Kirkham, Marson, \& Pearson, 2015), and 40,000 hospital admissions which represent $9.5 \%$ of all admissions for ACSCs (Dixon et al., 2015; Tian, Dixon, \& Gao, 2012). There are currently no published studies of care pathways for people after a suspected seizure (Scottish Ambulance Service, 2007a, 2007b). Paramedic practice in the National Health Service (NHS) in the UK is guided by UK Ambulance Services Clinical Practice Guidelines, published by the Joint Royal Colleges Ambulance Liaison Committee (JRCALC) and the Association of Ambulance Chief Executives (AACE) (JRCALC, 2013). The chapter on seizures focuses almost exclusively on rare medical emergencies and there is very little guidance on clinical assessment, risk stratification, non-conveyance or community management.
Suspected seizures are caused by a wide range of conditions, including cardiac or psychiatric disorders, which can mimic epileptic seizures (Malmgren, Reuber, \& Appleton, 2013), and emergency management decisions about these patients are complex and require expertise, training and guidance. In the UK, nearly all emergency calls for seizures lead to the dispatch of an ambulance, and a recent study in one UK ambulance study showed that $75 \%$ of these patients are transported to hospital (Dickson et al., 2015). Most suspected seizures have stopped by the time the ambulance or paramedics arrive on the scene (Burrell, Noble, \& Ridsdale, 2013; Dickson et al., 2017; O'Hara et al., 2015) and many of these patients do not require transport to hospital. 'Reducing the rate of ambulance 999 calls that result in transportation to A\&E' is an NHS Commissioning for Quality and Innovation (CQUIN) indicator (NHS England, 2016) and the ambulance service has tried to develop guidelines to support this aim. Emergency care pathways may allow the delivery of high quality care, without the need for transport to hospital (Snooks et al., 2014), but research is required to confirm their safety and effectiveness (Scottish Ambulance Service, 2007a; Snooks, 2004; Snooks et al., 2014).

An alternative care pathway (ACP) was set up by the Yorkshire Ambulance Service (YAS) in Doncaster, UK, in 2009. The ACP involved paramedics attending patients after a suspected seizure, and referring them to an epilepsy specialist nurse (ESN) service instead of conveying them to hospital. The ESN then contacted the patient within 48 hours, reviewed their history and considered what changes or investigations might be required as a result of the emergency incident. An initial evaluation of the 
Doncaster pathway showed that the satisfaction of those patients referred to the ACP was high and that the ESNs delivered meaningful interventions. However, the number of potential patients referred to the ACP was low and it was not possible to draw robust conclusions from the data. In response to these preliminary but positive data, a new ACP was established between YAS and Sheffield Teaching Hospitals NHS Foundation Trust (STH) in July 2015. Patients were eligible for the pathway if they lived within the geographical boundaries of two clinical commissioning groups: NHS Sheffield (460,326 adults, 569,737 adults and children) and NHS Rotherham (207,563 adults, 260,786 adults and children) (whose epilepsy services are provided by STH). This article reports on the results of an evaluation in the first year of the service (1 July 2015-31 May 2016).

\section{Methods}

\section{The alternative care pathway}

The ACP was aimed at adults ( $>16$ years old) with a known diagnosis of epilepsy. Known epilepsy was not strictly defined but it was intended to mean a diagnosis of epilepsy made by a doctor before the incident leading to the referral. The ACP had two elements. First, it provided a standard operating procedure and brief guidance for paramedics about which patients did not require conveyance to hospital. This guidance reiterated key phrases from the clinical practice guidelines but did not provide any additional decision support about conveyance. The second element was a mechanism of referral from paramedics in the community to the ESNs in STH. Paramedics referred patients by phoning the emergency operations centre (EOC) in YAS. Staff in the EOC, which is staffed 24 hours a day, would receive a verbal referral and complete all the relevant fields in the referral form which was then faxed to the ESN office at STH. The form included: referrer details, patient details, GP details, incident details (patient-reported medication, patient- or witness-reported seizure history and other medical history, paramedicreported injuries) and referral details (time and date). If the patient was under the care of a neurologist at STH, the ESNs would attempt to contact the patient by phone within five days of receiving the referral. If they were not under neurology care, but the ESNs thought that neurology review was necessary based on the information provided in the faxed referral, ESNs contacted the patient's GP to request a referral to a neurologist (this referral was required to comply with commissioning arrangements).

The ACP was launched with a two-hour educational event, which was attended by approximately 40 clinical staff and was delivered by the YAS paramedic lead for the ACP (TH). It was also disseminated using the weekly operational update, which is distributed to all clinical staff in YAS.

\section{Routine data collection from YAS}

YAS call handlers use advanced medical priority dispatch system (AMPDS) algorithms when receiving calls and making decisions about the type and urgency of response that is necessary. Suspected seizures are allocated AMPDS code 12. We used AMPDS code 12 to estimate the number of suspected seizure incidents in YAS during the study period. These data are collected routinely as part of the computer aided dispatch (CAD) dataset. It should be noted that the number of incidents and the number of patients are not the same. One patient may generate more than one incident over a period of time.

\section{Service evaluation methods, data collection and data analysis}

For each referral form received by the ESNs, a service evaluation form (SEF) was completed (Supplementary 1). Data from the YAS referral form and the STH SEF were entered into a Microsoft Excel ${ }^{\circledR}$ spreadsheet and analysed. Quantitative data were analysed using standard descriptive statistics. For patients who were referred multiple times during the evaluation, the most recent clinical and medical information is reported. Free text data on seizure frequency were transformed into a categorical variable with the following categories: daily, weekly, monthly, yearly and unknown.

The ESNs attempted to contact all patients and taking action was not dependent on a successful contact. For example, after failing to contact the patient by phone, they may have liaised with the patient's GP. We collected data on the actions/outcomes in the SEF, for example 'referred to the epilepsy clinic' or 'contacted pharmacist about anti-epileptic drugs'. These outcomes were categorised into positive or neutral by one of the authors (JMD). A positive outcome was one which was likely to result in improved patient care. A neutral outcome was one which was not likely to result in improvements in patient care but which was unlikely to cause any harm. Our dataset did not include a specific variable on adverse outcomes because these data are very difficult to collect without data linkage between organisations, but both YAS and STH were asked to report any adverse outcomes that were identified during operation of the ACP. We analysed data for referrals made during the first year of the service (1 July 201531 May 2016) (11 months).

\section{Ethics and approvals}

This project was registered and approved as a service evaluation by the Clinical Effectiveness Unit of STH (6896).

\section{Results}

YAS managed a total of 3964 suspected seizure incidents (AMDS code 12) in Sheffield (2687) and Rotherham 
(1277) during the study period, and an emergency vehicle arrived at the scene in $98.4 \%(3900 / 3964)$ of these incidents. During this period, paramedics made a total of 87 referrals to the ACP, which pertained to 74 individual patients $(87 / 3900 ; 2.2 \%)$ : three patients were referred twice, two patients were referred three times and two patients were referred four times.

From the 74 individual patients, 59.5\% (44/74) lived in Sheffield, 33.8\% (25/74) lived in Rotherham and 6.7\% (5/74) lived elsewhere. The median age was 41 (18-86) and $46.4 \%(32 / 74)$ were male. Of these patients, $52.7 \%$ (39/74) were under the care of a neurologist at STH (Sheffield or Rotherham). Using the patients' hospital records, we were able to confirm the seizure disorder diagnosis in $44.6 \%$ (33/74) of patients. From these 33 patients, $36.5 \%$ (27/33) had confirmed epilepsy, 5.5\% (4/33) had psychogenic non-epileptic seizures alone and 2.7\% (2/33) had comorbid epilepsy and psychogenic non-epileptic seizures. Of patients with confirmed epilepsy, $88.9 \%$ $(24 / 27)$ were taking at least one AED, with $33.3 \%(8 / 27)$ taking more than one AED. Of patients with confirmed epilepsy, 48.1\% (13/27) were having at least one seizure per month. Table 1 summarises the clinical characteristics of the cohort.

From the 87 individual referrals, 23\% (20/87) had suffered a seizure-related injury. It was possible to make contact with the patient after $73.6 \%(64 / 87)$ of the incidents: 21 patients could not be contacted, one was not contacted as the patient was already spoken to prior to receiving the referral form and the data for one patient were missing. The target of attempting to contact the patient within five days of receiving the referral was met in $97.3 \%(71 / 73)$ of incidents (missing data for contact time in 14/87). In both cases where the target was missed, patients were contacted within the five day target, leaving a phone message with patients returning the call later. After removing these two incidents, the median time to contact was 11 hours and the maximum was 4 days 19 hours. Once contacted, the median time spent talking to the patient on the phone was 10 minutes (range 5-30 mins). Table 2 summarises the activity of the epilepsy nurses after receiving the referral.

There was a positive action/outcome in 55\% (48/87) of incidents. Examples of positive outcomes included: contacting medical out-patients to arrange an appointment in the neurology clinic, contacting the patient's pharmacist to optimise AED therapy and booking the patient an appointment in the epilepsy nurse clinic. The nurses free hand notes give a flavour of these cases: 'the patient was treated for PNES, so a referral was made for the psychotherapy team', 'patient experiences regular seizures, and has never seen a neurologist, the patient was referred' and 'the patient has been encouraged to register with Sheffield GP so he can access services'. As far as we are aware, there were no adverse outcomes as a result of referral to the ACP.

\section{Discussion}

Our data show that an ACP for epileptic seizures has the potential to improve care for patients and to reduce unnecessary conveyance to hospital. Many of the patients referred to the ACP had a significant unmet need, principally poor seizure control, which the ESNs were able to take steps to address. Of the patients with a confirmed diagnosis of epilepsy, $48.1 \%$ were having at least one seizure per month. This may be due to sub-optimal treatment, or the ACP may have identified a population with

Table I. Seizure and medical characteristics of patients.

\begin{tabular}{|c|c|c|c|c|c|}
\hline Characteristic & Epilepsy & PNES & Both & $\begin{array}{l}\text { Unknown/could } \\
\text { not be confirmed }\end{array}$ & Total \\
\hline Patients $(n=74)$ & $36.5 \%(27)$ & $5.54 \%(4)$ & $2.7 \%(2)$ & $55.4 \%(4 I)$ & $100 \%(74)$ \\
\hline \multicolumn{6}{|l|}{$\operatorname{AEDs}(n=74)$} \\
\hline Yes & $88.9 \%(24)$ & $25 \%(I)$ & $100 \%(2)$ & $85.4 \%(35)$ & $83.8 \%(62)$ \\
\hline No & $3.7 \%(I)$ & $75 \%(3)$ & - & $12.2 \%(5)$ & $12.2 \%(9)$ \\
\hline Unknown & $7.4 \%(2)$ & - & - & $2.4 \%(I)$ & $4 \%(3)$ \\
\hline \multicolumn{6}{|l|}{ No. of AEDs } \\
\hline 1 & $50 \%(12)$ & - & - & $74.3 \%(26)$ & $61.2 \%(38)$ \\
\hline 2 & $20.8 \%(5)$ & - & $50 \%(I)$ & $8.6 \%(3)$ & $14.6 \%(9)$ \\
\hline 3 & $8.3 \%(2)$ & - & - & II.3\% (4) & $9.7 \%(6)$ \\
\hline 4 & $4.2 \%(I)$ & - & - & - & $1.6 \%(1)$ \\
\hline$\geq 5$ & - & - & - & $2.9 \%(I)$ & $1.6 \%(1)$ \\
\hline Unknown & $16.7 \%(4)$ & $100 \%(1)$ & $50 \%(I)$ & $2.9 \%(I)$ & $11.3 \%(7)$ \\
\hline \multicolumn{6}{|l|}{ Seizure frequency } \\
\hline Daily & $\mid 4.8 \%(4)$ & - & - & - & $5.4 \%(4)$ \\
\hline Weekly & | $4.8 \%(4)$ & - & - & $17 \%(7)$ & $14.9 \%(\mathrm{II})$ \\
\hline Monthly & $18.5 \%(5)$ & $25 \%(I)$ & $50 \%(I)$ & $19.5 \%(8)$ & $20.2 \%(15)$ \\
\hline Yearly & - & - & - & $9.8 \%(4)$ & $5.4 \%(4)$ \\
\hline Unknown & $51.9 \%(14)$ & $75 \%(3)$ & $50 \%(I)$ & $53.7 \%(22)$ & $54.1 \%(40)$ \\
\hline
\end{tabular}

AED: anti-epileptic drugs; PNES: psychogenic non-epileptic seizures. 
Table 2. Epilepsy specialist nurse activity/outcome after receiving referral from YAS.

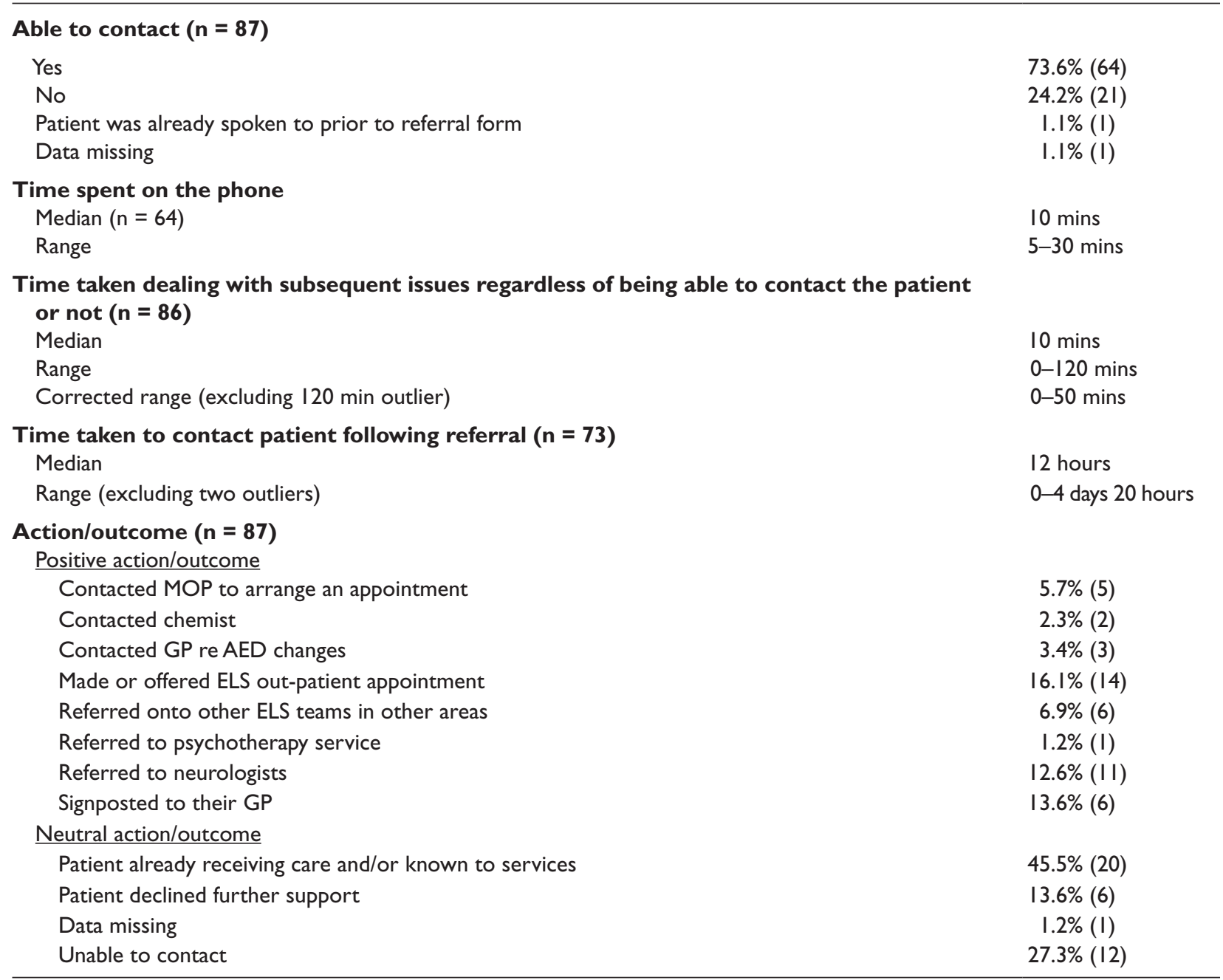

GP: general practitioner; ELS: epilepsy liaison service; OPA: out-patient appointment; re: regarding; MOP: medical out-patients.

treatment resistant epilepsy. Most of our patients were taking at least one AED, but it is not possible to determine if this was the correct treatment for them or to assess their adherence to their AED regime.

The additional workload for the ESNs during the study period was modest; they were able to comfortably accommodate the workload within their service, although ESN capacity would be an important consideration if the rate of referrals significantly increased. The ESNs were able to contact most patients quickly; the phone call for each case took a median time of 10 minutes (range 5-30 mins) and the subsequent work also took a median time of 10 minutes (range 0-50 mins). Telephone consultation allowed triage of patients' needs, and those patients who did not require any intervention could be managed without unnecessary hospital appointments.

Many patients do not receive the specialist follow-up that they need after a seizure in routine care in the NHS (Dixon et al., 2015), and our ACP was able to ensure that these patients received an enhanced level of care. The ACP gave rise to a positive outcome in $55 \%$ of cases.
Although we categorised the actions of the ESNs as positive or neutral, these were not true outcomes and our categorisation of the action the ESNs took after the telephone call was retrospective and based on free text entries by the nurses. A weakness of the current data is that we did not formally measure whether the actions taken by the ESNs had a measurable effect on patients' subsequent seizures or other relevant outcomes (such as healthcare utilisation behaviour) or patients' satisfaction with the ACP. An area which requires further work is the development of success criteria for the ACP which can be applied to future evaluations.

Effective implementation of the ACP was impeded by NHS commissioning arrangements. Under local agreements, a patient who has been referred by their GP to the epilepsy clinic within the last two years may be assessed/ treated by STH without a new referral. For all other patients, a new referral from the GP is required before that patient may be assessed/treated. Of the 74 patients referred to our ACP, $39(52.7 \%)$ patients were under the care of a neurologist at STH and $33(44.6 \%)$ were not. This meant 
that the only option for the 33 patients not under neurology care was a letter to the GP asking him/her to consider referral (this restriction does not apply to direct referral from A\&E). This arrangement, which is in place to ensure that the hospital trust is paid appropriately for seeing patients, is a significant barrier to the effective working of the pathway and new commissioning arrangements are an important area for further development of this pathway. The fact that the majority of patients in this study were under the care of a neurologist is in contrast to the general population where only a minority are under neurology care (Reuber, Torane, \& Mack, 2010). This discrepancy is probably a reflection of the fact that patients with epilepsy under the care of a neurologist tend to have more severe epilepsy and/or that a criterion for referral was that the patient had 'known epilepsy'.

Our data show that only $2.2 \%$ (87/3900) of YAS emergency incidents for seizures were referred to the ACP during the study period. In the EPIC studies (Dickson et al., 2015; Dickson et al., 2017), 74.2\% of AMPDS code 12 incidents were thought to be seizures by paramedics on the scene after clinical assessment. Of these, $13.2 \%$ were a first epileptic seizure, $30.8 \%$ were an epileptic seizure (known diagnosis of epilepsy), 20.9\% were an epileptic seizure (not first seizure, no formal epilepsy diagnosis) and $9.9 \%$ were an acute symptomatic seizure. Therefore, 891 patients $((3900 \times 74.2 \%) \times 30.8 \%)$ were potentially eligible for the ACP but only $9.8 \%$ (87/891) were referred. To make a significant impact on avoidable attendances/ admissions, a much higher referral rate would be required. So why was the referral rate so low?

Several published studies have shown that paramedics do not feel confident or supported to make decisions about non-conveyance after a seizure. A UK-based qualitative study of decision-making in emergency ambulance clinicians identified themes such as lack of experience, patient views, insufficient information, anxiety over litigation, lack of access to the patients' medical records and bystander expectation as factors influencing the rate of transfer to hospital in this group of patients (Burrell et al., 2013). Many paramedics feel transport to hospital is both clinically safer and a lower risk medico-legally. Other qualitative studies have looked at decision making in paramedics in other patient populations and they have studied institutional and organisational barriers to non-conveyance and other behavioural changes. But there are no published articles systematically looking at barriers and facilitators to implementation of ACPs in pre-hospital care.

Current UK ambulance service guidelines state that 'known epileptics who make a full recovery, are not at risk and can be supervised adequately, can be managed at home following local guidelines' (JRCALC, 2013). This statement is the only guidance that is provided and it does not encourage or support non-conveyance. Our ACP did not provide any additional guidance to support paramedics in identifying patients that were safe to be left at the scene. Such a decision support tool is likely to be necessary to guide paramedics when making these difficult decisions. Examples of these include accepting mild tachycardia as part of the normal post-ictal phase and finding ways to stop this automatically triggering transport to hospital as a consequence of a raised early warning score, agreeing criteria for 'appropriate supervision' by a carer during recovery and allowing transport home for patients who have a seizure away from home such as in a local shop or on the street.

A significant challenge in this ACP and in its future development is the requirement for inter-organisational collaborations. YAS covers a large geographical area and not all of these areas have mature epilepsy services with the capacity to offer a phone call with ESNs. Even if capacity did exist within epilepsy services, identifying service managers, who are already busy with existing services, to drive the proposed change remains a significant challenge.

\section{Conclusion}

An ACP for people after a suspected seizure has the potential to safely reduce rates of transport to hospital and to improve care for people with epilepsy. However, paramedics in our study used the ACP for only a small proportion of those patients who were potentially eligible. Further research is required to develop tools to support paramedics to confidently identify patients that are suitable for management without transport to hospital.

\section{Author contributions}

$\mathrm{TH}, \mathrm{CM}$ and RAG were responsible for implementation of the clinical service. JMD proposed conducting the service evaluation, created the SEF and co-ordinated data collection and analysis. KM collected and collated the data. GHR analysed the data. JMD, RAG and MR provided academic oversight throughout the service evaluation. All authors have read and approved the manuscript. We would like to acknowledge the important contribution of all the epilepsy specialist nurses in STH and the paramedics and other staff at YAS, especially Trevor Baldwin who provided YAS CAD data for the study.

\section{Conflict of interest}

None declared.

\section{Funding}

None.

\section{References}

Bardsley, M., Blunt, I., Davies, S., \& Dixon, J. (2013). Is secondary preventive care improving? Observational study of 10-year trends in emergency admissions for conditions amenable to ambulatory care. BMJ Open, 3, e002007. 
Burrell, L., Noble, A., \& Ridsdale, L. (2013). Decision-making by ambulance clinicians in London when managing patients with epilepsy: a qualitative study. Emergency Medical Journal, 30, 236-240.

Dickson, J., Taylor, L., Shewan, J., Baldwin, T., Grünewald, R., \& Reuber, M. (2015). A cross-sectional study of the prehospital management of adult patients with a suspected seizure (EPIC1). BMJ Open, 6, e010573.

Dickson, J., Dudhill, H., Shewan, J., Mason, M., Grünewald, R., \& Reuber, M. (2017). Cross-sectional study of the hospital management of adult patients with a suspected seizure (EPIC2). BMJ Open, 7, e015696.

Dixon, P., Kirkham, J., Marson, A., \& Pearson, M. (2015). National audit of seizure management in hospitals (NASH): results of the national audit of epilepsy in the UK. BMJ Open, 5, e007325.

Joint Royal Colleges Ambulance Liaison Committee (JRCALC). (2013). UK ambulance services clinical practice guidelines 2013. Bristol: Class Professional Publishing.

Malmgren, K., Reuber, M., \& Appleton, R. (2013). Differential diagnosis of epilepsy. In S. Shorvon, R. Guerrini, M. Cook, \& S. Lhatoo (Eds.), Oxford textbook of epilepsy and epileptic seizures (pp. 81-94). Oxford: Oxford University Press.

Manjunath, R., Paradis, P., Parise, H., Lafeuille, M., Bowers, B., Duh, M., ... Faught, E. (2012). Burden of uncontrolled epilepsy in patients requiring an emergency room visit or hospitalization. Neurology, 79, 1908-1916.

NHS England. (2016). Commissioning for quality and innovation (CQUIN) guidance for 2016/17. Retrieved from https://www.england.nhs.uk/wp-content/ uploads/2016/03/cquin-guidance-16-17-v3.pdf.

O'Hara, R., Johnson, M., Siriwardena, A. N., Weyman, A. Turner, J., Shaw, D., ... Shewan, J. (2015). A qualitative study of systemic influences on paramedic decision making: care transitions and patient safety. Journal of Health Services Research and Policy, 20, 45-53.

Reuber, M., Torane, P., \& Mack, C. (2010). Do older adults have equitable access to specialist epilepsy services? Epilepsia, 51, 2341-2343.

Ridsdale, L., Virdi, C., Noble, A., \& Morgan, M. (2012). Explanations given by people with epilepsy for using emergency medical services: a qualitative study. Epilepsy and Behaviour, 25, 529-533.

Scottish Ambulance Service. (2007a). A review of Scottish Ambulance Service see and treat guidelines. Scottish Ambulance Service.

Scottish Ambulance Service. (2007b). Treat and refer guidelines: do we like them? Do we use them? Scottish Ambulance Service.

Snooks, H., Carter, B., Dale, J., Foster, T., Humphreys, I., Logan, P., ... Sanchez, A. (2014). Support and assessment for fall emergency referrals (SAFER 1): cluster randomised trial of computerised clinical decision support for paramedics. PLoS ONE, 9, e106436.

Snooks, H. A. (2004). On-scene alternatives for emergency ambulance crews attending patients who do not need to travel to the accident and emergency department: a review of the literature. Emergency Medicine Journal, 21, 212-215.

Tian, Y., Dixon, A., \& Gao, H. (2012, April). Emergency hospital admissions for ambulatory care-sensitive conditions: identifying the potential for reductions. Report for the King's Fund. https://www.kingsfund.org. uk/sites/default/files/field/field_publication_file/ data-briefing-emergency-hospital-admissionsfor-ambulatory-care-sensitive-conditions-apr2012.pdf 